\title{
Cucurbit[8]uril-Regulated Colloidal Dispersions Exhibiting Photocontrolled Rheological Behavior **
}

\author{
Chi Hu, Ji Liu, Yuchao Wu, Kevin R. West, Oren A. Scherman *
}

Abstract: In-situ photocontrol over shear-thickening of condensed colloidal dispersions is of paramount importance in a wide range of applications including process technology and photorheological fluids. Its development and practicability, however, are hampered by the lack of well-designed photoresponsive systems. Here, we report a colloidal suspension whose rheological behavior is readily switchable between shear-thinning and shear-thickening using an external light stimulus. This smart colloidal solution contains hybrid raspberry-like colloids prepared by employing cucurbit[8]uril as a supramolecular linker to assemble functional $\mathrm{Fe}_{3} \mathrm{O}_{4}$ nanoparticles onto a silica core. The formed raspberry colloids are photoresponsive and can be reversibly disassembled under UV irradiation.

There has been a growing interest in designing fluids whose rheological properties can be tuned by external light stimuli, i.e., photorheological fluids. ${ }^{[1-5]}$ In particular, light-induced switching between shear-thinning and shearthickening behavior in colloidal suspensions has proven to be useful in a number of applications. ${ }^{[6]}$ For example, many colloidal dispersions in paints, coatings and lubricants exhibit a rapid, sometimes discontinuous increase in viscosity with small increases in shear rate when subjected to rapid shear during processing. In such cases, shear thickening can be detrimental and should be minimized or eliminated, as it can damage processing equipment and induce dramatic changes in the dispersion microstructure, which results in poor product quality. ${ }^{[6]}$ On the other hand, shear-thickening effects can be exploited in the design of protection devices such as body armor. Here the fluid can provide a self-

\footnotetext{
** This research has been supported by the Engineering Physical Science Research Council (EPSRC, grant EP/K028510/1), and the Natural Science Foundation of Jiangsu Province (grant SBK2017041610). C.Hu thanks BP for financial support.

* Dr. C. Hu,

Department of Pharmaceutical Engineering

China Pharmaceutical University

Nanjing 210009 (China)

Dr. J. Liu, Y. Wu, Prof. O.A. Scherman

Melville Laboratory for Polymer Synthesis

Department of Chemistry, University of Cambridge

Cambridge, CB2 1EW (UK)

E-mail: oas23@cam.ac.uk

Dr. K.R. West

BP Oil UK Ltd

Whitchurch Hill, Pangbourne, Reading

Berkshire, RG8 7QR (UK)
}

Supporting information for this article is available on the WWW under http://www.your.website.com or from the author. limiting maximum rate of flow through a highly nonlinear response. ${ }^{[7]}$ Despite the technological importance of controlling this phenomenon, there has been little effort to explore readily switchable shear-thinning and shear-thickening fluids using an external stimulus such as light.

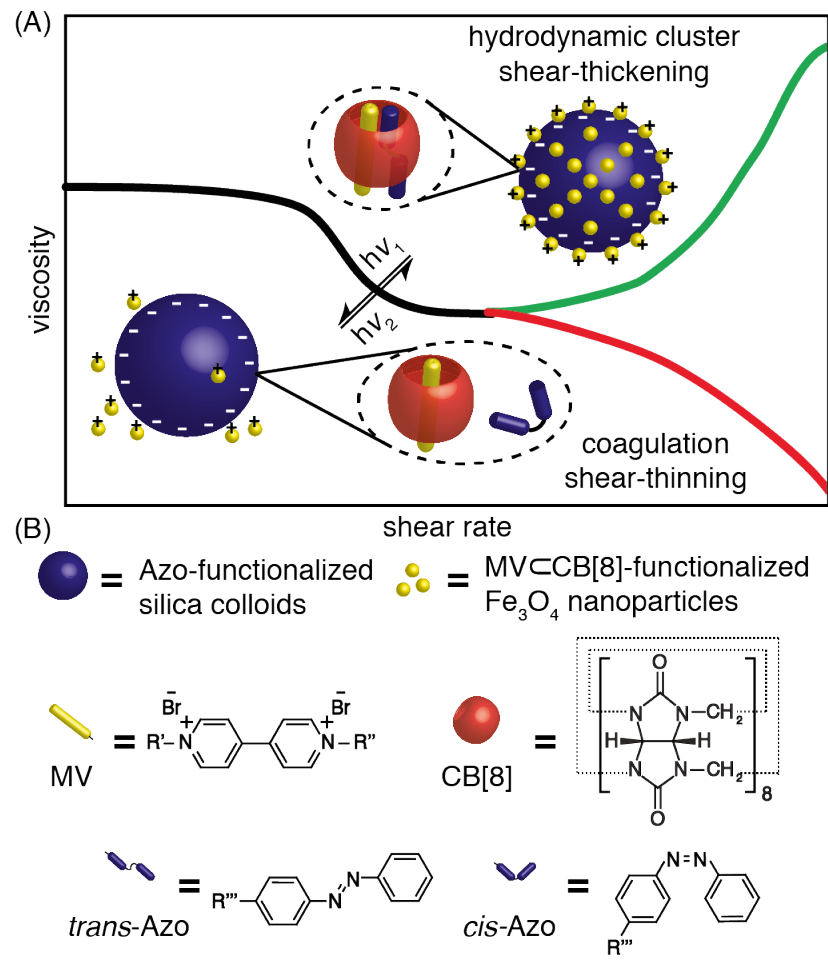

Figure 1. (A) Schematic rheological behavior of a light-induced reversible assembly of raspberry-like colloids. At low shear rates, the fluid's viscosity drops, displaying what is known as shear thinning (black curve). Conversely, at high shear rates, assembled raspberry colloids exhibit repulsive surface interactions and form hydrodynamic clusters under shear, resulting in a shear-thickening phenomenon (green curve). Disassembled raspberry colloids exhibit attractive interparticular forces and coagulate under shear, giving rise to a shear-thinning effect (red curve). (B) Functional components used to assemble hybrid raspberry colloids with $\mathrm{CB}[8]$ as a supramolecular linker, where R' $=-\mathrm{CH}_{3}, \mathrm{R}^{\prime \prime}=-\left(\mathrm{CH}_{2}\right)_{2} \mathrm{OCONH}\left(\mathrm{CH}_{2}\right)_{3} \mathrm{Si}\left(\mathrm{OCH}_{2} \mathrm{CH}_{3}\right)_{3}$ and $\mathrm{R} "=-\mathrm{OCONH}\left(\mathrm{CH}_{2}\right)_{3} \mathrm{Si}\left(\mathrm{OCH}_{2} \mathrm{CH}_{3}\right)_{3}$.

Photoacid generators are a class of commercially available molecules that can be photolyzed by UV light to produce an acidic moiety. They have attracted the attention of several researchers for the development of photorheological fluids. ${ }^{[8-10]}$ Raghavan and co-workers developed a system consisting of disk-like nanoparticles of laponite with a surfactant stabilizer and a photoacid generator. Upon light activation, the nanoparticles assembled into a physical network 


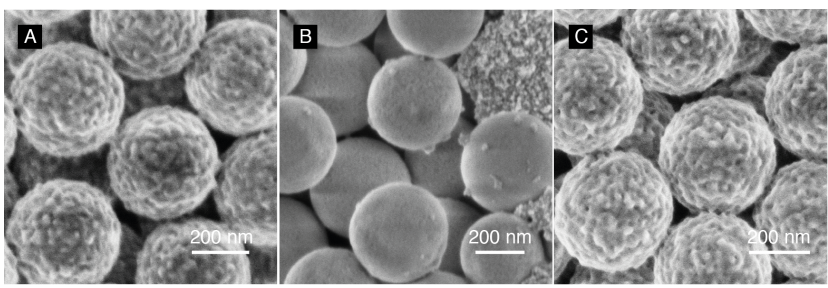

Figure 2. SEM images of (A) assembled raspberry colloids through $\mathrm{CB}$ [8]-mediated supramolecular chemistry; (B) disassembled raspberry colloids and (C) re-assembled raspberry colloids. All samples were spun cast onto a glass slide.

(gel). ${ }^{[5]}$ Several years later, the same group reported a lightactivated ionic gelation of alginate and pectin through addition of salt as well as a photoacid generator to the biopolymers in an aqueous solution. ${ }^{[11]}$ Moreover, reversible photoacids, characterized by a proton dissociation process, have been studied and utilized to trigger gelation and obtain control over the mechanical properties of gels. ${ }^{[12]}$ In most cases, however, the system maintains the gel state if kept under acidic $\mathrm{pH}$, which can be unfavorable for certain applications. ${ }^{[13]}$ Eelkema and van Esch demonstrated spatial control over the self-assembly of molecular fibers through the use of a photoacid, where materials remained in the gel state across the entire $\mathrm{pH}$ range. ${ }^{[14]}$ This example, however, lacks reversibility of the gel formation and exhibits poor control over the mechanical properties of the material.

Cucurbit[8]uril (CB[8]) is a macrocyclic host molecule, formed by eight glycoluril repeating units linked together through methylene bridges with a symmetric "barrel" shape. ${ }^{[15-18]} \mathrm{CB}[8]$ can simultaneously accommodate two guest molecules, e.g., an electron-deficient first guest viologen (MV) and an electron-rich second guest azobenzene (Azo) yielding a stable 1:1:1 heteroternary complex through multiple and synergistic noncovalent interactions. ${ }^{[19-30]} \mathrm{We}$ recently reported the preparation of photoresponsive hybrid raspberry-like colloids based on the host-guest interactions of $\mathrm{CB}[8] .^{[31-34]}$ The application of supramolecular systems to tune interparticular forces and rheological behavior of the resulting colloidal dispersion, however, remains unexplored. ${ }^{[35-37]}$

Herein, we present a facile route to prepare raspberrylike colloids. Small $\mathrm{MV}$-functionalized $\mathrm{Fe}_{3} \mathrm{O}_{4}$ nanoparticles are assembled with supramolecular linker $\mathrm{CB}$ [8] onto a larger silica core which has Azo derivatives on its periphery (Figure 1). Moreover, the non-covalent nature of $\mathrm{CB}$ [8] affords the assembly of raspberry colloids with lightcontrolled reversibility. The assembled raspberry colloids exhibit repulsive surface forces on account of MV dications on the surface. They form hydrodynamic clusters under high shear rates, resulting in a shear-thickening effect. On the other hand, the disassembled system contains negatively-charged silica colloids and positively charged $\mathrm{Fe}_{3} \mathrm{O}_{4}$ nanoparticles, which are attractive to each other and coagulate under shear, giving rise to a shear-thinning phenomenon. In this system, a light stimulus controls both the assembly of raspberry colloids as well as rheological properties of the resulting colloidal suspension. The results show for the first time that shear thinning and shear thickening can be readily accessed in an efficient and controllable fashion, giving rise to potential applications in various processing techniques and shock-absorption applications (e.g., body armor). For example, the raspberry colloid containing fluid could control damping by being switched between shearthickened and flowing states using external light irradiation.

Hybrid raspberry-like colloids were assembled by adding an aqueous dispersion of Azo-silica colloids into a suspension of the $\mathrm{MV}-\mathrm{Fe}_{3} \mathrm{O}_{4}$ nanoparticles. The latter were precomplexed with an excess amount of $\mathrm{CB}[8]$ in water, forming a heteroternary complex of $(\mathrm{MV} / \mathrm{Azo}) \subset \mathrm{CB}[8]$ at the particle-particle interface (see reference 34 and ESI for detailed information). After $5 \mathrm{~min}$ of sonication, hybrid colloids with a well-defined raspberry-like architecture could be clearly observed by TEM imaging as shown in Figure S5, where $\mathrm{Fe}_{3} \mathrm{O}_{4}$ nanoparticles are densely packed on the periphery of the silica colloids. The uniform structure of the raspberry colloids is confirmed by SEM (Figure 2A) and the diameter corresponds to the $\mathrm{D}_{T E M}$ of $396 \mathrm{~nm}$ as calculated from Figure S5.

As schematically shown in Figure 1, the supramolecular self-assembly of raspberry colloids is a light-driven reversible process, which is controlled by the molecular recognition of $\mathrm{CB}$ [8] with two different Azo photoisomers in particular. The extended trans-Azo structure can be encapsulated into the cavity of $\mathrm{CB}[8]$ together with MV to form a heteroternary complex of (MV/trans-Azo) C CB[8]. However, complexation of the photogenerated, bent cis-Azo isomer inside the $\mathrm{CB}[8]$ cavity with $\mathrm{MV}$ is geometrically unfavorable. ${ }^{[38]}$ The formed raspberry colloids can be reversibly disassembled under UV light irradiation $(350 \mathrm{~nm}$ for $10 \mathrm{~min}$ ), which triggers the trans to cis isomerization of Azo derivatives leading to dissociation of the heteroternary complex (MV/trans-Azo) $\subset \mathrm{CB}[8]$, thereby eliminating the supramolecular linkage at the particle-particle interface (Figure 2B). The detached raspberry colloids can be reassembled under visible light (420 $\mathrm{nm}$ for $10 \mathrm{~min})$, where cis-Azo is transformed to the trans isomer, reforming the heteroternary complexes (MV/Azo) $\subset \mathrm{CB}[8]$ as supramolecular linkers between the silica core and $\mathrm{Fe}_{3} \mathrm{O}_{4}$ nanoparticle corona. The SEM image in Figure $2 \mathrm{C}$ shows the raspberrylike morphology of the reassembled hybrid colloids.

During the light-controlled reversible assembly of raspberry colloids, not only does the morphology of the particles change, but also their surface interactions and therefore their rheological properties. As shown by the black line in Figure $3 \mathrm{~A}$, assembled raspberry colloids exhibit a zeta potential of $+17 \mathrm{mV}$, thus displaying a repulsive interparticular force in solution. As a result, this raspberry colloidal dispersion fits into the general jamming paradigm in soft matter at high particle concentrations ( $\geq 60 \mathrm{vol} \%$ ), where non-Newtonian behavior is observed, and the fluid's viscosity depends on an applied shear stress or shear rate. ${ }^{[39]}$ At low shear rates, the fluid's viscosity drops in a response known as shear thinning, 

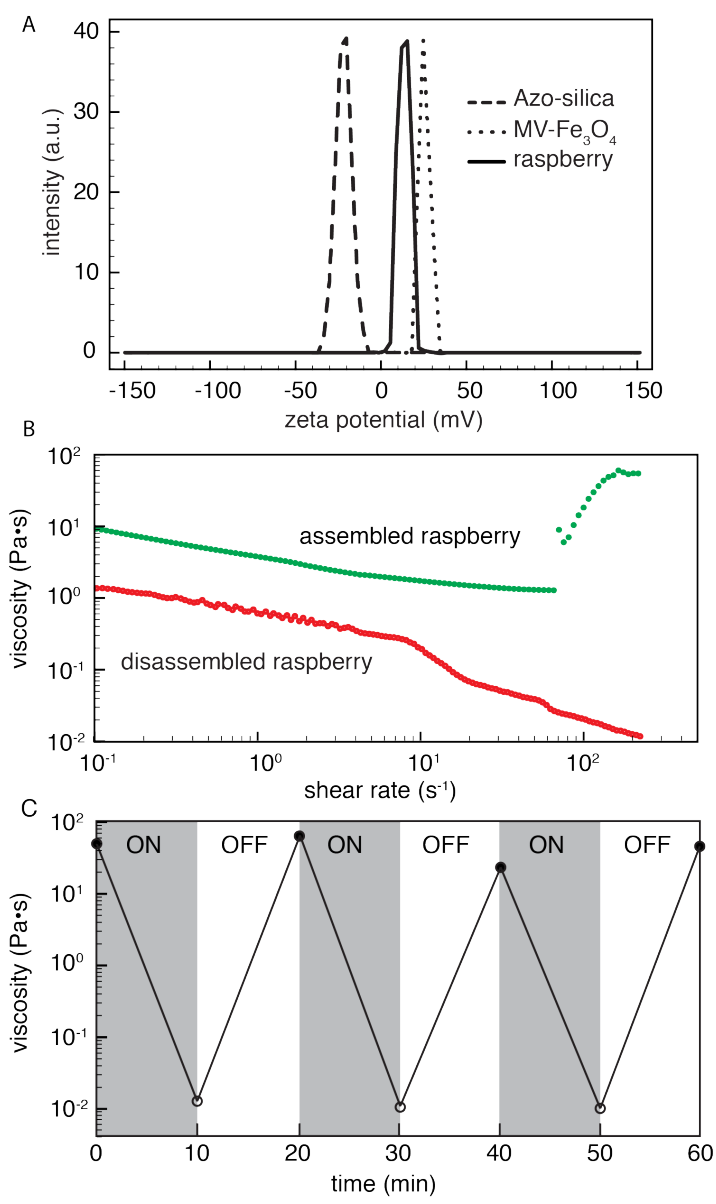

Figure 3. Effects of the photogenerated changes in the surface forces and the rheological properties of the raspberry colloidal solution. (A) Zeta potential of Azo-silica colloids, $\mathrm{MV}-\mathrm{Fe}_{3} \mathrm{O}_{4}$ nanoparticles and the hybrid raspberry colloids at $\mathrm{pH}$ 7. (B) Viscosity as a function of shear rate for assembled raspberry colloids (green dots) and disassembled colloids (red dots) at $60 \%$ volume fraction and $\mathrm{pH} 7$. (C) Viscosity at a shear rate of $200 \mathrm{~s}^{-1}$ during three cycles of UV and visible light irradiation.

where flowing particles become organized and lower their viscosity. ${ }^{[40]}$ At high shear rates, the formation of jammed clusters bound together by hydrodynamic lubrication forces prevents the particles to flow around each other. This results in a higher rate of energy dissipation and abrupt or even discontinuous increase in viscosity once a critical shear rate is reached. ${ }^{[41-43]}$ As shown in the steady shear measurement (green line in Figure 3B), the dynamic viscosity of the raspberry colloidal suspension increases from 2 to $10 \mathrm{~Pa} \cdot \mathrm{s}$ as the applied shear rate reaches $80 \mathrm{~s}^{-1}$ on account of the formation of hydrodynamic clusters.

Once the critical shear rate is reached, hydrodynamic interactions between particles dominate over stochastic ones, and the microstructures in the colloidal dispersion become significantly different from the seemingly random microstructures observed near equilibrium. Particles aggregate into closely-connected hydroclusters, which are driven by transient concentration fluctuations and sustained by the applied shear field, manifesting greater anisotropy in the microstructure. This observation is consistent with previ- ous work on the effect of interparticular forces in shearthickening colloidal dispersions. ${ }^{[4]}$ When the $\mathrm{pH}$ is adjusted away from the isoelectric point, the repulsive forces between the particles decrease the viscosity at low/intermediate shear rates; however, the viscosity increases at or just after a critical shear rate providing that the volume fraction of solids in the dispersion is sufficiently high. ${ }^{[45,46]}$ As the isoelectric point is the $\mathrm{pH}$ value at which the zeta potential is approximately zero, the system reported here (at $\mathrm{pH} 7$ and a zeta potential of $+17 \mathrm{mV}$ ) is clearly away from its isoelectric point, making it suitable to exhibit repulsive interparticular forces and demonstrate shear-thickening behavior. Indeed, in typical colloidal solutions the separation between hydroclustered particles is predicted to be on the order of nanometers, so their shear-thickening behavior directly reflects the particles' surface structure and any short-range interparticular forces present in the system. The formulated dispersion can be exploited depending on the specific processes or applications desired by manipulating the interparticular forces.

On the contrary, after UV irradiation the disassembled raspberry colloids only show a shear-thinning phenomenon (red dots in Figure 3B). In water, the silanol-OH groups on the surface of the silica colloids react with $\mathrm{H}^{+}$at low $\mathrm{pH}$ or $\mathrm{OH}^{-}$at high $\mathrm{pH}$, creating either positive $\left(\mathrm{SiOH}_{2}{ }^{+}\right)$or negative $\left(\mathrm{SiO}^{-}\right)$surface charges, respectively. The isoelectric point of the silanol -OH groups on the silica colloids is at $\mathrm{pH} 2$, where the majority of surface sites are neutral $(\mathrm{SiOH})$ and the net charge on the colloid surface is zero. ${ }^{[47]}$ In our system, the $\mathrm{pH}$ was adjusted to 7 by adding $\mathrm{HCl}$ or $\mathrm{NaOH}$, thus the Azo-silica colloids were negatively charged and displayed a zeta potential of $-25 \mathrm{mV}$ (dashed line in Figure 3A). Native $\mathrm{Fe}_{3} \mathrm{O}_{4}$ nanoparticles possess negative surface charges and show a zeta potential of $-40 \mathrm{mV}$ at $\mathrm{pH} 7$, however, they became positively charged with a zeta potential of $+28 \mathrm{mV}$ after functionalization with MV (dotted line in Figure 3A). As shear thickening occurs only when there is no overall attraction between the particles, ${ }^{[4,48-50]}$ the attractive interactions between Azo-silica colloids and $\mathrm{MV}-\mathrm{Fe}_{3} \mathrm{O}_{4}$ nanoparticles give rise to the formation of coagulation instead of hydrodynamic clusters, thus inhibiting the occurrence of shear thickening. Several cycles of reversible assembly of raspberry colloids were carried out by alternating UV/visible light irradiation, and the viscosity at a shear rate of $200 \mathrm{~s}^{-1}$ shows a clear switching behavior without any obvious decay (Figure 3C).

Figure 4 shows the solid loading dependencies of the apparent transition from shear thinning to shear thickening when the shear rate exceeds a critical level. Shear thickening (solid symbols in Figure 4) is present in all the concentrated colloidal dispersions regardless of the particle volume fraction. A precise determination of the onset critical shear rate can be obtained as the dilatant transition is rather abrupt. The shear-thickening transition occurs at a shear rate of $10 \mathrm{~s}^{-1}$ for the highest concentrated suspension with a solids loading of $65 \mathrm{vol} \%$, at $80 \mathrm{~s}^{-1}$ for the dispersion having a particle volume fraction of $60 \mathrm{vol} \%$ and at $200 \mathrm{~s}^{-1}$ 


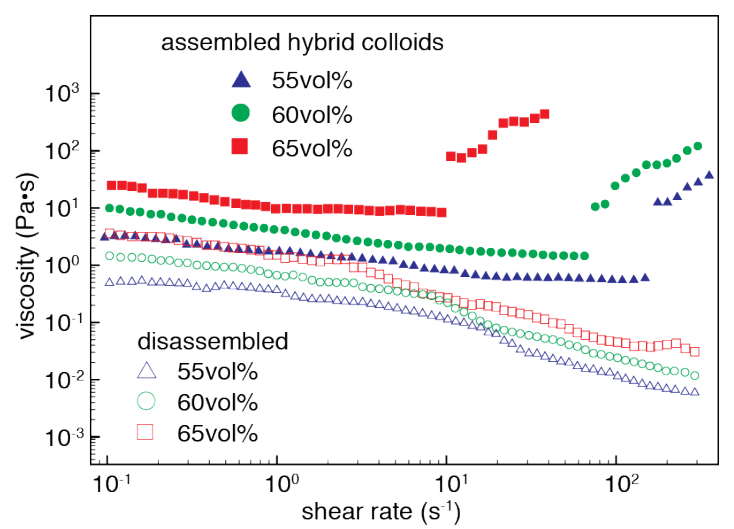

Figure 4. Photoresponsive viscosity-shear rate under steady shear flow for 55,60 and $65 \%$ volume fraction colloidal dispersion in water. Shear thickening and thinning behavior are represented by solid symbols and empty symbols, respectively.

where the particle fraction is only 55 vol\%. This suggests that the critical shear rate decreases with increased particle volume fraction. At a higher solid loading, particles collide more frequently with each other yielding hydroclusters and result in transient fluctuations of particle concentration. In the shear-thickening regime at high shear rates, the higher solids-loading dispersions (60 vol\% and 65 vol\%) exhibit greater viscosity compared to the lower particle volume fraction solutions ( $55 \mathrm{vol} \%$ ).

While assembled hybrid raspberry-like colloids exhibit both shear-thinning and shear-thickening behavior, a shearthinning regime was only observed for the aqueous dispersions containing disassembled hybrid colloids across a wide range of solids loadings. Suspensions containing either 65 vol\% or 60 vol\% disassembled colloids exhibited increased viscosity over the 55 vol\% dispersion over the entire shear rate range investigated (empty symbols in Figure 4), in agreement with the general trend for the assembled hybrid raspberry-like colloids.

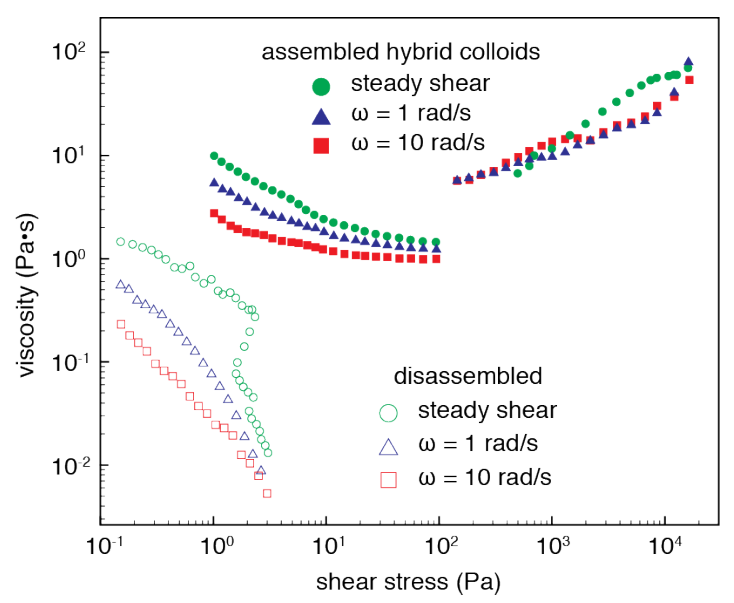

Figure 5. Light-controlled shear-thickening phenomena of 60 vol\% hybrid raspberry-like colloids dispersed in water for both steady and dynamic shear plotted against the average applied dynamic shear stress.

The complex viscosity as a function of average applied dynamic shear stress for the aqueous colloidal dispersion at $60 \mathrm{vol} \%$ is presented in Figure 5, together with the steady shear viscosity as a function of steady shear stress for comparison. A shear-thickening transition was observed in both steady and dynamic measurements (the solid symbols in Figure 5). The complex viscosity was characterized for both ascending and descending stress sweeps (see Figure S8), confirming the reversibility of the shear-thickening effect. Although shear thickening is a stress-controlled behavior, steady shear viscosity and complex dynamic viscosity do not overlay when plotted against applied stress. ${ }^{[40]}$ Dynamic viscosity is found to be comparable to the steady shear viscosity measured at the same average applied stress at or after the point of shear thickening. Deviation from the steady and dynamic viscosities is evident especially at low shear stress (Figure 5), where increased angular frequency affords decreased dynamic viscosity in both the assembled and disassembled hybrid raspberry-like colloids.

The empirical Cox-Merz rule clearly does not hold for this system, which is a non-Newtonian fluid. ${ }^{[51]}$ However, the Delaware-Rutgers rule suggests that slowly relaxing materials respond to highest shear rate during a dynamic measurement. Therefore, dynamic and steady properties overlay when the highest shear rate experienced during the oscillation is taken as the effective steady shear rate. ${ }^{[52]}$ The Delaware-Rutgers rule explains the deviation observed in the shear-thinning regime for the $60 \mathrm{vol} \%$ colloidal dispersion, however, it does not explore the shear-thickening transition or the shear-thickened state. The response of this material to shear stress is in a nonlinear regime and the shear-thickening transition is relatively fast. This result is consistent with previous work by Wagner, ${ }^{[40]}$ Raghavan and Khan, ${ }^{[53]}$ and Mewis and Biebaut, ${ }^{[54]}$ who reported disagreement between the Delaware-Rutgers rule and their experimental data upon shear thickening.

The $\mathrm{MV}-\mathrm{Fe}_{3} \mathrm{O}_{4}$ nanoparticles were detached from the Azo-silica core under UV light. The disassembled particle mixture shows entirely different rheological behavior when plotted against the average applied dynamic shear stress in both steady and dynamic shear measurements. Disassembled hybrid colloids exhibit shear thinning over the entire range of stress investigated and show much lower viscosity compared to the assembled hybrid colloids (empty symbols in Figure 5). This is on account of the attraction between the positively-charged $\mathrm{MV}-\mathrm{Fe}_{3} \mathrm{O}_{4}$ nanoparticles and the negatively-charged Azo-silica colloids, which gives rise to precipitation under shear.

In summary, we have demonstrated the ability to photocontrol the assembly of hybrid raspberry-like colloids in a reversible supramolecular fashion. $\mathrm{Fe}_{3} \mathrm{O}_{4}$ nanoparticles were bound to the periphery of silica colloids through the formation of heteroternary complexes (MV/Azo) C CB[8], which act as supramolecular linkers at the particle-particle interface. The non-covalent nature of $\mathrm{CB}$ [8] host-guest interactions afford the assembly of raspberry colloids and their light-controlled reversibility. Most importantly, we 
were able to tune the interparticular forces through the reversible assembly of raspberry colloids using UV/visible light irradiation. The resulting morphological changes on the nanoscale have a dramatic impact on the rheological behavior of the colloidal solution. For the first time, shear-thinning and shear-thickening colloidal dispersions can be reversibly switched in situ upon exposure to an external stimulus.

\section{References}

[1] W. M. Winslow, J. Appl. Phys. 1949, 20, 1137-1140.

[2] B. Jr., S. Dyke, M. Sain, J. Carlson, J. Eng. Mech. 1997, 123, 230238.

[3] A. M. Ketner, R. Kumar, T. S. Davies, P. W. Elder, S. R. Raghavan, J. Am. Chem. Soc. 2007, 129, 1553-1559.

[4] R. Kumar, A. M. Ketner, S. R. Raghavan, Langmuir 2010, 26, 54055411.

[5] K. Sun, R. Kumar, D. E. Falvey, S. R. Raghavan, J. Am. Chem. Soc. 2009, 131, 7135-7141.

[6] J. Bender, N. J. Wagner, J. Rheol. 1996, 40, 899-916.

[7] Y. Lee, E. D. Wetzel, N. J. Wagner, J. Mater. Sci. 2003, 38, 28252833.

[8] M. Shirai, M. Tsunooka, Prog. Polym. Sci. 1996, 21, 1-45.

[9] W. Zhou, S. M. Kuebler, K. L. Braun, T. Yu, J. K. Cammack, C. K. Ober, J. W. Perry, S. R. Marder, Science 2002, 296, 1106-1109.

[10] J. L. Dektar, N. P. Hacker, J. Org. Chem. 1990, 55, 639-647.

[11] V. Javvaji, A. G. Baradwaj, G. F. Payne, S. R. Raghavan, Langmuir 2011, 27, 12591-12596.

[12] Z. Shi, P. Peng, D. Strohecker, Y. Liao, J. Am. Chem. Soc. 2011, 133, 14699-14703.

[13] J. Raeburn, T. O. McDonald, D. J. Adams, Chem. Commun. 2012 48, 9355-9357.

[14] C. Maity, W. E. Hendriksen, J. H. vanEsch, R. Eelkema, Angew. Chem. Int. Ed. 2015, 54, 998-1001.

[15] J. Kim, I.-S. Jung, S.-Y. Kim, E. Lee, J.-K. Kang, S. Sakamoto, K Yamaguchi, K. Kim, J. Am. Chem. Soc. 2000, 122, 540-541.

[16] A. Day, A. P. Arnold, R. J. Blanch, B. Snushall, J. Org. Chem. 2001, 66, 8094-8100.

[17] S. Liu, C. Ruspic, P. Mukhopadhyay, S. Chakrabarti, P. Y. Zavalij, L. Isaacs, J. Am. Chem. Soc. 2005, 127, 15959-15967.

[18] J. Liu, C. S. Y. Tan, Y. Lan, O. A. Scherman, Macromol. Chem. Phys. 2015, 217, 319-332.

[19] H.-J. Kim, J. Heo, W. S. Jeon, E. Lee, J. Kim, S. Sakamoto, K. Yamaguchi, K. Kim, Angew. Chem. Int. Ed. 2001, 40, 1526-1529.

[20] C. Hu, F. Tian, Y. Zheng, C. S. Y. Tan, K. R. West, O. A. Scherman, Chem. Sci. 2015, 6, 5303-5310.

[21] C. Hu, Y. Lan, F. Tian, K. R. West, O. A. Scherman, Langmuir 2014, 30, 10926-10932.

[22] H. Yang, B. Yuan, X. Zhang, O. A. Scherman, Accounts Chem. Res. 2014, 47, 2106-2115.

[23] C. Stoffelen, J. Voskuhl, P. Jonkheijm, J. Huskens, Angew. Chem. Int. Ed. 2014, 53, 3400-3404.
[24] X. Yan, F. Wang, B. Zheng, F. Huang, Chem. Soc. Rev. 2012, 41, 6042-6065.

[25] C. Márquez, R. R. Hudgins, W. M. Nau, J. Am. Chem. Soc. 2004, $126,5806-5816$

[26] J. Liu, C. S. Y. Tan, Z. Yu, Y. Lan, C. Abell, O. A. Scherman, Adv. Mater. 2017, 29, 1604951.

[27] J. Liu, C. S. Y. Tan, Z. Yu, N. Li, C. Abell, O. A. Scherman, Adv. Mater. 2017, 29, 1605325.

[28] C. Hu, Y. Lan, K. R. West, O. A. Scherman, Adv. Mater. 2015, 27, 7957-7962.

[29] C. Hu, Y. Zheng, Z. Yu, C. Abell, O. A. Scherman, Chem. Commun. 2015, 51, 4858-4860.

[30] J. Liu, Y. Lan, Z. Y. Yu, C. S. Y. Tan, R. M. Parker, C. Abell, O. A. Scherman, Acc. Chem. Res. 2017, 50, 208-217.

[31] Y. Lan, Y. Wu, A. Karas, O. A. Scherman, Angew. Chem. Int. Ed. 2014, 53, 2166-2169.

[32] X. Lu, E. Masson, Langmuir 2011, 27, 3051-3058.

[33] C. Kim, S. S. Agasti, Z. Zhu, L. Isaacs, V. M. Rotello, Nat. Chem. 2010, 2, 962-966.

[34] C. Hu, K. R. West, O. A. Scherman, Nanoscale 2016, 8, 7840-7844.

[35] Q. Song, Y. Jiao, Z. Wang, X. Zhang, Small 2016, 12, 24-31.

[36] X. Yan, D. Xu, X. Chi, J. Chen, S. Dong, X. Ding, Y. Yu, F. Huang, Adv. Mater. 2012, 24, 362-369.

[37] X. Ji, B. Shi, H. Wang, D. Xia, K. Jie, Z. L. Wu, F. Huang, Adv. Mater. 2015, 27, 8062-8066.

[38] F. Tian, D. Jiao, F. Biedermann, O. A. Scherman, Nat. Commun. 2012, 3, 1207.

[39] A. J. Liu, S. R. Nagel, Nature 1998, 396, 21-22.

[40] Y. Lee, N. Wagner, Rheol. Acta 2003, 42, 199-208.

[41] N. J. Wagner, J. F. Brady, Phys. Today 2009, 62, 27-32.

[42] R. L. Hoffman, J. Rheol. 1998, 42, 111-123.

[43] X. Cheng, J. H. McCoy, J. N. Israelachvili, I. Cohen, Science 2011, 333, 1276-1279.

[44] G. V. Franks, Z. Zhou, N. J. Duin, D. V. Boger, J. Rheol. 2000, 44, 759-779.

[45] J Vermant, M. J. Solomon, J. Phys.: Condens. Matter 2005, 17, 187216.

[46] B. V. Velamakanni, J. C. Chang, F. F. Lange, D. S. Pearson, Langmuir 1990, 6, 1323-1325.

[47] E. Matijević, R. James, G. Parks in Surface and Colloid Science, Vol. 12, Springer US, 1982, pp. 119-216.

[48] H. A. Barnes, J. Rheol. 1989, 33, 329-366.

[49] H. Freundlich, H. L. Roder, Trans. Faraday Soc. 1938, 34, 308-316.

[50] J. R. V. Wazer, E. Besmertnuk, J. Phys. Colloid Chem. 1950, 54, 89106.

[51] W. P. Cox, E. H. Merz, J. Polym. Sci. 1958, 28, 619-622.

[52] D. Doraiswamy, A. N. Mujumdar, I. Tsao, A. N. Beris, S. C. Danforth, A. B. Metzner, J. Rheol. 1991, 35, 647-685.

[53] S. R. Raghavan, S. A. Khan, J. Colloid Interface Sci. 1997, 185, 57 67.

[54] J. Mewis, G. Biebaut, J. Rheol. 2001, 45, 799-813. 


\section{Entry for the Table of Contents (Please choose one layout only)}

Layout 1:

Supramolecular Self-assembly

Chi Hu, Ji Liu, Yuchao Wu, Kevin R. West, Oren A. Scherman

Cucurbit[8]uril-Regulated Colloidal Dispersions Exhibiting Photocontrolled Rheological Behavior

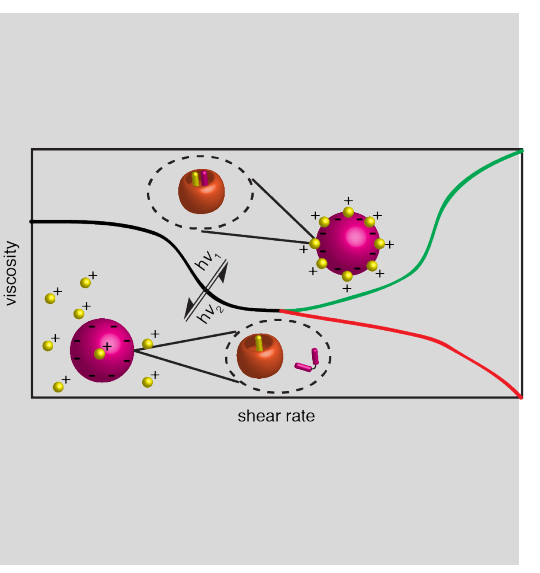

Photoresponsive reversible shear thickening in raspberry-like colloidal dispersion: Hybrid raspberrylike colloids composed of $\mathrm{Fe}_{3} \mathrm{O}_{4}$ corona nanoparticles and silica core were assembled using cucurbit[8]uril as a supramolecular linker. The light-controlled reversibility of this assembly process gives rise to fine control over interparticle forces and the resulting rheological properties.

Layout 2:

Catch Phrase:

Author(s), Corresponding Author(s)* . Page Page

Title Text
((The TOC Graphic should not exceed the size of this area))

Text for Table of Contents, max. 450 characters. 